\title{
Comunicação
}

\section{Metodologia para estimar o número de dias trabalháveis com máquinas agrícolas}

\author{
Javier Solis Estradal*, José Fernando Schlosser², Marcelo Silveira de Farias ${ }^{3}$, \\ Gustavo Oliveira do Santos ${ }^{4}$, Iury Yago Port Rüdell ${ }^{5}$
}

http://dx.doi.org/10.1590/0034-737X201562040011

\begin{abstract}
RESUMO
No atual modelo de agricultura, é indispensável que o produtor conheça a real disponibilidade de tempo para a execução das operações agrícolas ao longo do ciclo das culturas. Este é o primeiro passo para se planejar e se obter maior eficiência na execução dessas operações, que estão sujeitas às variações do clima. As variáveis meteorológicas de uma determinada região influenciam diretamente no número de dias disponíveis para trabalhar com máquinas agrícolas. O objetivo deste trabalho foi estimar as probabilidades de ocorrência de dias favoráveis para o trabalho mecanizado, utilizando-se informações meteorológicas do município de Santa Maria, RS, Brasil. As condições para se considerar o dia como favorável para o trabalho com máquinas foram a precipitação $<5 \mathrm{~mm}$ e o armazenamento de água no solo (ARM) entre 40 e 90\% da capacidade de água disponível (CAD). O método da cadeia de Markov de primeira ordem foi utilizado para estimar as probabilidades condicionais de dias favoráveis ao trabalho com máquinas. Os resultados indicam que a metodologia aplicada para estimar as probabilidades de dias favoráveis ao uso de máquinas agrícolas foi viável, evidenciando as épocas mais apropriadas à execução de operações agrícolas mecanizadas no campo, para o município de Santa Maria, RS.
\end{abstract}

Palavras-chave: gestão de máquinas agrícolas, probabilidade de dias úmidos, cadeia de markov.

\section{ABSTRACT}

\section{Methodology to estimate the number of workable days with farm machinery}

In the current agricultural model, it is essential for farmers to know the real time available for the execution of agricultural operations throughout the crop cycle. This is the first step to plan and achieve greater efficiency in these operations, which are subject to climate changes. Meteorological variables in a certain region directly influence the number of days available to work with farm machinery. The objective of this study was to estimate the probabilities of favorable workdays for farm machinery, using meteorological information from the municipality of Santa Maria, RS, Brazil. The conditions to be considered as a favorable working day with machinery were: precipitation $<5 \mathrm{~mm}$ and soil water storage between 40 and $90 \%$ of the available water capacity. The first order Markov chain was used to estimate the conditional probabilities of favorable days to work with machines. Results indicate that the methodology used to estimate the probabilities of favorable working days for agricultural machinery was feasible, showing the most appropriate times to execution of mechanized farming operations in the field, in the municipality of Santa Maria, RS.

Key words: agricultural machinery management, probability of wet days, markov chain.

\footnotetext{
Submetido em 19/11/2014 e aprovado em 26/06/2015

${ }^{1}$ Universidade Federal de Santa Maria, Departamento de Engenharia Rural, Santa Maria, Rio Grande do Sul, Brasil. j.solis.estrada@gmail.com

${ }^{2}$ Universidade Federal de Santa Maria, Departamento de Engenharia Rural, Santa Maria, Rio Grande do Sul, Brasil. josefernandoschlosser@gmail.com

${ }_{3}^{3}$ Universidade Federal de Santa Maria, Departamento de Engenharia Rural, Santa Maria, Rio Grande do Sul, Brasil. silveira_farias@hotmail.com

${ }^{4}$ Universidade Federal de Santa Maria, Departamento de Engenharia Rural, Santa Maria, Rio Grande do Sul, Brasil. gustavo_santos_rs@hotmail.com

${ }^{5}$ Universidade Federal de Santa Maria, Santa Maria, Rio Grande do Sul, Brasil. iuryrudell22@gmail.com

*Autor para correspondência: j.solis.estrada@gmail.com
} 


\section{INTRODUÇÃO}

Os sistemas de produção agrícola têm sofrido alterações com o passar dos anos, transformando-se em um modelo empresarial. A competitividade imposta à agricultura exige do produtor rural a racionalização dos recursos, dentre eles, os mecanizados, no sentido de aumentar a produtividade e diminuir os custos de produção (Hunt, 2008).

Por essas razões, é indispensável que o produtor conheça a real disponibilidade de tempo para a execução das operações agrícolas. Este é o primeiro passo para se planejar e se obter eficiência na execução dessas operações, visto que as atividades agrícolas estão sujeitas às variações do clima.

As variáveis meteorológicas influenciam o número de dias disponíveis para trabalhar com máquinas agrícolas (Ataíde et al., 2012). Precipitações prolongadas e intensas, segundo Araujo-Junior et al. (2011), causam dificuldades nas operações de campo, diminuindo o rendimento das máquinas.

A viabilidade das operações com máquinas agrícolas, desde o preparo do solo até a colheita, depende da dinâmica da água nas camadas superficiais do solo, que serve como um indicador para a tomada de decisão (Fernandes et al, 2000).

Neste sentido, há uma demanda crescente, por parte dos produtores rurais, de trabalhos de pesquisa, no sentido de melhorar a eficiência das atividades com máquinas agrícolas, visto que em solos com excesso de umidade as máquinas trabalham com dificuldade e, além disso, essa umidade concorre para a compactação do solo, não se conseguindo um trabalho satisfatório e degradando a qualidade física do solo (Roque et al., 2011; Braunack et al., 2006; Hemmat et al., 2009).

Com o propósito de determinar o número de dias trabalháveis com máquinas agrícolas, diferentes municípios do Brasil vêm sendo pesquisados. A região central do Rio Grande do Sul, contudo, ainda não foi estudada. A metodologia utilizada baseia-se na análise de variáveis locais, principalmente, a precipitação e o armazenamento de água no solo (ARM), aliados à aplicação do modelo da cadeia de Markov (Assis et al., 1989). A aplicação desse modelo, de primeira ordem, baseia-se na probabilidade de um dado evento ocorrer, dado o evento que ocorreu no dia anterior.

Este trabalho foi conduzido com o objetivo de estimar as probabilidades de ocorrência de dias favoráveis para o trabalho com máquina agrícola, utilizando-se informações meteorológicas do município de Santa Maria, RS, Brasil.

\section{MATERIAL E MÉTODOS}

Foram utilizados dados meteorológicos diários de precipitação $(\mathrm{mm})$ e temperatura mínima e máxima $\left({ }^{\circ} \mathrm{C}\right)$, referentes ao período de 1993 a 2013, obtidos do banco de dados do Instituto Nacional de Meteorologia (INMET), na área de abrangência da Estação Meteorológica Principal de Santa Maria. A área estudada localizase entre as coordenadas de $29,7^{\circ} \mathrm{Sul}, 53,7^{\circ}$ Oeste, $95 \mathrm{~m}$ altitude, abrangendo uma superfície total de $6.640 \mathrm{~km}^{2}$ (Cardoso, 2005). O solo do município pertence à unidade de mapeamento Santa Maria, classificado como Argissolo Vermelho distrófico arênico, segundo classificação da Embrapa (2006), por ser o solo mais representativo $(42,69 \%)$ na área de abrangência do estudo (Cardoso, 2005).

Calculou-se o balanço hídrico sequencial diário de acordo com a metodologia descrita por Thornthwaite \& Mather (1955), utilizando-se, como capacidade de água disponível (CAD), o valor de $100 \mathrm{~mm}$, em função das características físicas do solo da região. As condições para se considerar o dia como favorável ao trabalho de máquinas agrícolas são a precipitação < $5 \mathrm{~mm}$ e o ARM entre 40 e $90 \%$ da CAD (Erthal \& Sentelhas, 1995; Aviad et al., 2009).

Com base nas duas condições estabelecidas, foi elaborada uma planilha eletrônica no programa informático Microsoft Excel $^{\circledR}$, baseada no método da cadeia de Markov para calcular, apresentar e discutir a determinação das probabilidades simples e condicionais, visando à estimativa da ocorrência de dias considerados favoráveis $(\mathrm{F})$, ou, não favoráveis $(\mathrm{NF})$ ao trabalho de máquinas agrícolas (Assis et al., 1989).

a) Probabilidades simples:

$$
\begin{aligned}
& \mathrm{P}(\mathrm{NF})=\mathrm{NF} / \text { TotalNF; } \\
& \mathrm{P}(\mathrm{F})=\mathrm{F} / \text { TotalF; }
\end{aligned}
$$

b) Probabilidades condicionais:

$$
\begin{aligned}
& \mathrm{P}(\mathrm{NF} / \mathrm{NF})=(\mathrm{NF} / \mathrm{NF}) / \mathrm{TNF} ; \\
& \mathrm{P}(\mathrm{F} / \mathrm{F})=(\mathrm{F} / \mathrm{F}) / \mathrm{TF} ; \\
& \mathrm{P}(\mathrm{F} / \mathrm{NF})=1-\mathrm{P}(\mathrm{NF} / \mathrm{NF}) ; \\
& \mathrm{P}(\mathrm{NF} / \mathrm{F})=1-\mathrm{P}(\mathrm{F} / \mathrm{F}),
\end{aligned}
$$

em que: $\mathrm{P}(\mathrm{NF})$ é a probabilidade de um dia não favorável, no decêndio considerado; $\mathrm{P}(\mathrm{F})$ é a probabilidade de um dia ser favorável, no decêndio considerado; $\mathrm{P}(\mathrm{NF} /$ NF) a probabilidade de um dia não ser favorável, dado que o anterior também não foi favorável ao trabalho com máquinas; $\mathrm{P}(\mathrm{F} / \mathrm{F})$ a probabilidade de um dia ser favorável, dado que o anterior foi favorável; $\mathrm{P}(\mathrm{F} / \mathrm{NF})$ a probabilidade de um dia ser favorável, dado que o anterior foi não favorável e $\mathrm{P}(\mathrm{NF} / \mathrm{F})$ é a probabilidade de um dia não ser favorável, dado que o anterior foi favorável. 


\section{RESULTADOS E DISCUSSÃO}

A Tabela 1 apresenta os valores das probabilidades de ocorrência de dias favoráveis $(\mathrm{F})$, não favoráveis $(\mathrm{NF})$ e suas respectivas probabilidades condicionais para o trabalho com máquinas agrícolas no município de Santa Maria, RS, no período de 1993 a 2013.

Para esse município, os valores de $\mathrm{P}(\mathrm{F})$ variaram entre $11 \%$ (no segundo e terceiro decêndio de junho) e $74 \%$ (segundo decêndio de fevereiro), sendo que o valor médio para o período foi equivalente a $38 \%$, com desvio padrão de $19 \%$. Já para as $\mathrm{P}(\mathrm{F} / \mathrm{NF})$, os valores variaram entre 3\% (terceiro decêndio de junho) e 67\% (segundo decêndio de janeiro).

Com base nessa informação, o período ótimo para as operações agrícolas estende-se desde o segundo decêndio de novembro até os primeiros dez dias de abril. Este período foi determinado por causa de os valores médios de $\mathrm{P}(\mathrm{F})$ e $\mathrm{P}(\mathrm{F} / \mathrm{F})$ serem maiores que $50 \%$, para este intervalo de tempo (Tabela 1). Ao longo desses meses, apenas o segundo decêndio de dezembro e o primeiro decêndio de março apresentaram valores de $\mathrm{P}(\mathrm{F})$ inferiores a $50 \%$.

Resultados semelhantes para valores de $\mathrm{P}(\mathrm{F})$ foram encontrados por Monteiro et al (2014), ao estimarem as probabilidades de ocorrência de dias favoráveis ao tráfego de máquinas agrícolas, para o município de Passo Fundo, RS, onde a variação de $\mathrm{P}(\mathrm{F})$ esteve entre $14 \%$, no primeiro decêndio de julho, e $62 \%$, no segundo decêndio de março, considerando-se uma probabilidade média de 38\% no período entre 1979 e 2008.

Tabela 1: Probabilidades de ocorrência de dias favoráveis, não favoráveis e suas respectivas probabilidades condicionais em Santa Maria, RS (1993 - 2013)

\begin{tabular}{|c|c|c|c|c|c|c|c|}
\hline Decêndio & Dias & $\mathbf{P}(\mathbf{F})$ & $\mathbf{P}(\mathrm{NF})$ & $\mathbf{P}(\mathrm{NF} / \mathrm{NF})$ & $\mathbf{P}(\mathrm{F} / \mathrm{NF})$ & $\mathbf{P}(\mathbf{F} / \mathbf{F})$ & $P(N F / F)$ \\
\hline Jan1 & 10 & 0,53 & 0,47 & 0,53 & 0,47 & 0,57 & 0,43 \\
\hline Jan2 & 10 & 0,64 & 0,36 & 0,33 & 0,67 & 0,76 & 0,24 \\
\hline Jan3 & 11 & 0,63 & 0,37 & 0,53 & 0,47 & 0,76 & 0,24 \\
\hline Fev1 & 10 & 0,58 & 0,42 & 0,51 & 0,49 & 0,67 & 0,33 \\
\hline Fev2 & 10 & 0,74 & 0,26 & 0,37 & 0,63 & 0,78 & 0,22 \\
\hline Fev3 & 8 & 0,52 & 0,48 & 0,60 & 0,40 & 0,69 & 0,31 \\
\hline Mar1 & 10 & 0,46 & 0,54 & 0,57 & 0,43 & 0,53 & 0,47 \\
\hline Mar2 & 10 & 0,64 & 0,36 & 0,52 & 0,48 & 0,71 & 0,29 \\
\hline Mar3 & 11 & 0,70 & 0,30 & 0,43 & 0,58 & 0,81 & 0,19 \\
\hline Abr1 & 10 & 0,55 & 0,45 & 0,57 & 0,43 & 0,68 & 0,32 \\
\hline Abr2 & 10 & 0,39 & 0,61 & 0,61 & 0,39 & 0,53 & 0,47 \\
\hline Abr3 & 10 & 0,52 & 0,48 & 0,56 & 0,44 & 0,59 & 0,41 \\
\hline Mai1 & 10 & 0,40 & 0,60 & 0,69 & 0,31 & 0,61 & 0,39 \\
\hline Mai2 & 10 & 0,46 & 0,54 & 0,76 & 0,24 & 0,58 & 0,42 \\
\hline Mai3 & 11 & 0,34 & 0,66 & 0,74 & 0,26 & 0,52 & 0,48 \\
\hline Jun1 & 10 & 0,19 & 0,81 & 0,90 & 0,10 & 0,20 & 0,80 \\
\hline Jun2 & 10 & 0,11 & 0,89 & 0,93 & 0,07 & 0,18 & 0,82 \\
\hline Jun3 & 10 & 0,11 & 0,89 & 0,97 & 0,03 & 0,20 & 0,80 \\
\hline Jul1 & 10 & 0,14 & 0,86 & 0,94 & 0,06 & 0,27 & 0,73 \\
\hline Jul2 & 10 & 0,14 & 0,86 & 0,96 & 0,04 & 0,26 & 0,74 \\
\hline Jul3 & 11 & 0,12 & 0,88 & 0,95 & 0,05 & 0,27 & 0,73 \\
\hline Ago1 & 10 & 0,16 & 0,84 & 0,91 & 0,09 & 0,22 & 0,78 \\
\hline Ago2 & 10 & 0,26 & 0,74 & 0,84 & 0,16 & 0,35 & 0,65 \\
\hline Ago3 & 11 & 0,32 & 0,68 & 0,84 & 0,16 & 0,48 & 0,52 \\
\hline Set1 & 10 & 0,39 & 0,60 & 0,68 & 0,32 & 0,39 & 0,61 \\
\hline Set2 & 10 & 0,23 & 0,77 & 0,81 & 0,19 & 0,27 & 0,73 \\
\hline Set3 & 10 & 0,26 & 0,74 & 0,86 & 0,14 & 0,37 & 0,63 \\
\hline Out1 & 10 & 0,31 & 0,69 & 0,83 & 0,17 & 0,48 & 0,52 \\
\hline Out2 & 10 & 0,34 & 0,66 & 0,81 & 0,19 & 0,60 & 0,40 \\
\hline Out3 & 11 & 0,41 & 0,59 & 0,66 & 0,34 & 0,54 & 0,46 \\
\hline Nov1 & 10 & 0,45 & 0,55 & 0,61 & 0,39 & 0,54 & 0,46 \\
\hline Nov2 & 10 & 0,57 & 0,43 & 0,57 & 0,43 & 0,74 & 0,26 \\
\hline Nov3 & 10 & 0,57 & 0,43 & 0,48 & 0,52 & 0,59 & 0,41 \\
\hline Dez1 & 10 & 0,59 & 0,41 & 0,48 & 0,52 & 0,66 & 0,34 \\
\hline Dez2 & 10 & 0,45 & 0,55 & 0,59 & 0,41 & 0,54 & 0,46 \\
\hline Dez3 & 11 & 0,52 & 0,48 & 0,54 & 0,46 & 0,61 & 0,39 \\
\hline
\end{tabular}

Rev. Ceres, Viçosa, v. 62, n.4, p. 410-414, jul-ago, 2015 
A maior probabilidade de ocorrência de dias favoráveis ao trabalho com máquinas agrícolas, para o município de Santa Maria, em relação à probabilidade de dias favoráveis para o município de Passo Fundo, pode ser atribuída às características físico-hídricas do solo. Segundo Kaufmann et al (2012), em Latossolos, solo característico do município de Passo Fundo, alterações na disponibilidade de água no solo podem ser intensificadas pela dispersão da argila na superfície. A liberação de argila reduz a dimensão dos canalículos do solo pela iluviação de partículas finas, criando um selamento na superfície do solo (Lima Neto et al., 2010).

Ao observar a Tabela 1, verificam-se duas situações opostas. A probabilidade de um dia ser favorável, dado que o anterior foi favorável $\mathrm{P}(\mathrm{F} / \mathrm{F})$, é maior que a probabilidade simples de ter um dia favorável ao trabalho com máquinas. Já os valores da probabilidade de um dia ser favorável, dado que o anterior foi não favorável $\mathrm{P}(\mathrm{F} / \mathrm{NF})$, são inferiores aos $\mathrm{P}(\mathrm{F})$. As probabilidades refletem a dependência das condições do dia anterior. Ao realizar um estudo semelhante para o município de Londrina-PR, Ataíde et al. (2012) concluíram que existe uma forte dependência de ocorrer um dia favorável, se este for imediatamente precedido por um dia favorável, e vice-versa.

Os valores mostrados na Tabela 1 podem ser usados para identificar os meses associados com maior probabilidade de ocorrer dias não favoráveis para operações com máquinas agrícolas no campo.

De acordo com a Figura 1, pode-se observar que no município de Santa Maria, há uma precipitação frequente ao longo do ano, com média mensal superior aos 100 $\mathrm{mm}$. O total de precipitação média anual do período estudado foi de $1.771 \mathrm{~mm}$. A precipitação pluvial anual média em todo o Rio Grande do Sul é de $1.500 \mathrm{~mm}$ (Britto et al., 2008). A maior e a menor precipitação médias mensais observadas foram, respectivamente, 192,18 mm, em outubro, e 119,60 mm, em agosto (Figura 1).

Os meses do ano mais favoráveis para o uso de máquinas agrícolas no campo são determinados pela precipitação média do município. O conhecimento dos períodos mais favoráveis é considerado em conjunção com o do teor de umidade do solo, para se determinarem os períodos de semeadura, colheita e uso racional de agrotóxicos (Peloia \& Milan, 2010).

No município de Santa Maria, como as chuvas são bem distribuídas durante o ano, diferentemente de outros municípios brasileiros, chovendo cerca de $150 \mathrm{~mm}$ por mês, a ocorrência de dias favoráveis à mecanização verificou-se durante os meses de novembro e abril, graças às menores precipitações médias (Figura 1).

Na Tabela 2, apresentam-se as probabilidades médias de ocorrência de dias favoráveis e suas respectivas probabilidades condicionais, para as principais operações agrícolas realizadas durante o ciclo da cultura do arroz irrigado, no município de Santa Maria, RS.

Observa-se que durante o último trimestre do ano e o primeiro do seguinte, foram obtidas as maiores quantidades de dias disponíveis para trabalho com máquinas agrícolas. A informação sobre esse período é importante para o planejamento das operações de semeadura, tratamentos fitossanitários, colheita e preparo antecipado do solo (Tabela 2). Corroborando essa informação, recomenda-se que, para o município de Santa Maria, a semeadura ocorra entre 01 de outubro e 10 de dezembro (Embrapa, 2007).

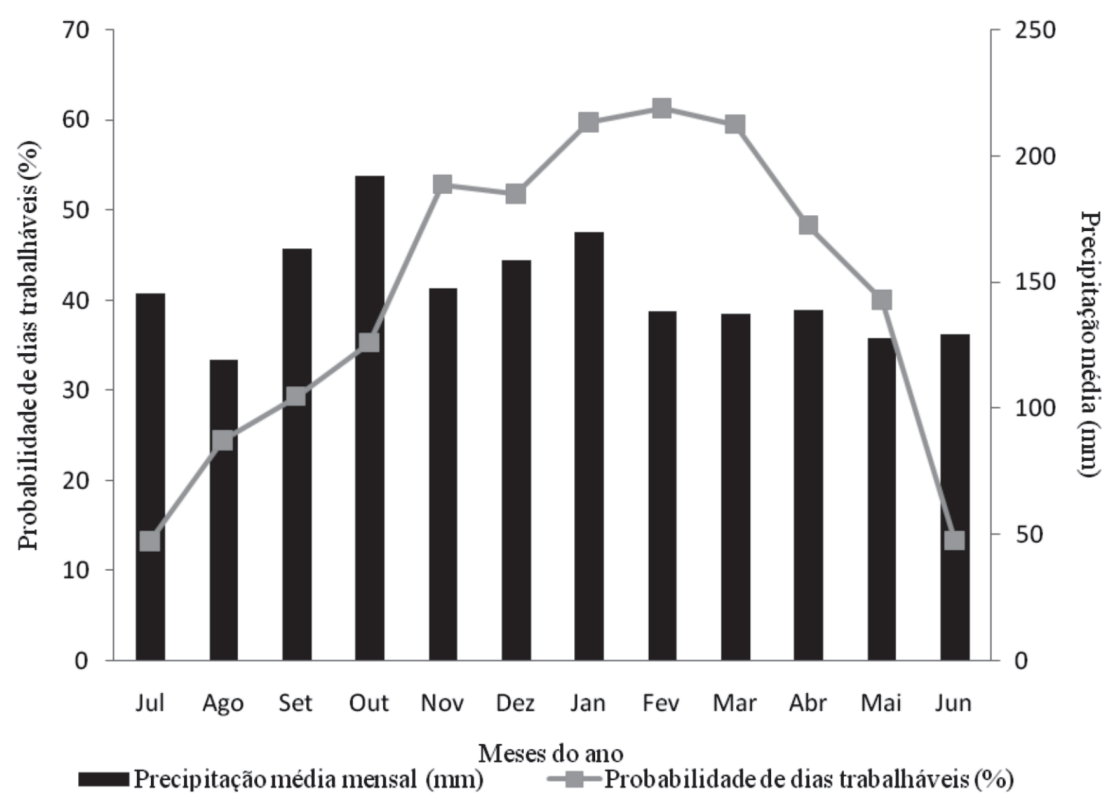

Figura 1: Precipitação média mensal e probabilidades médias de ocorrência de dias trabalháveis em Santa Maria, RS (1993 - 2013). 
Para a cultura do arroz irrigado, Marchezan (1994) indica que as operações de preparo de solo são realizadas entre os meses de agosto e setembro, seguidas de um período de 30 a 60 dias de pousio, para que ocorra a reinfestação das áreas com plantas daninhas. Esse período, indicado pelo autor, coincide com os trimestres com as menores probabilidades de dias disponíveis para o trabalho com máquinas agrícolas (Tabela 2). Isso explica os atrasos que ocorrem durante a realização dessas operações e justifica a importância da realização do preparo antecipado do solo, logo após o término da colheita.

Tabela 2: Probabilidades médias de ocorrência de dias favoráveis e suas respectivas probabilidades condicionais para as operações agrícolas da cultura do arroz irrigado, no município de Santa Maria, RS

\begin{tabular}{|c|c|c|c|c|}
\hline \multirow{2}{*}{ Trimestre } & \multicolumn{3}{|c|}{ Probabilidades médias simples e condicionais } & \multirow{2}{*}{$\begin{array}{c}\text { Operações agrícolas } \\
\text { Arroz irrigado }\end{array}$} \\
\hline & $\mathrm{P}(\mathrm{F})$ & $\mathrm{P}(\mathrm{F} / \mathrm{F})$ & $\mathrm{P}(\mathrm{NF} / \mathrm{F})$ & \\
\hline 1 & 0,60 & 0,70 & 0,30 & TF; C; PAS \\
\hline 2 & 0,34 & 0,45 & 0,55 & C; PAS \\
\hline 3 & 0,22 & 0,32 & 0,68 & PS \\
\hline 4 & 0,47 & 0,59 & 0,41 & $\mathrm{~S} ; \mathrm{TF}$ \\
\hline
\end{tabular}

C - Colheita; PAS - Preparo antecipado do solo; PS - Preparo do solo; S - Semeadura; TF - Tratamento fitossanitário.

\section{CONCLUSÕES}

O modelo aplicado para estimar a probabilidade de dias favoráveis ao uso de máquinas agrícolas foi aprovado, uma vez que evidenciou as épocas mais apropriadas à execução de operações agrícolas mecanizadas, para o município de Santa Maria, RS.

O período favorável para o trabalho com máquinas agrícolas, nesse município, é compreendido entre o segundo decêndio de novembro e o primeiro decêndio de abril.

\section{REFERÊNCIAS}

Araujo-Junior CF, Dias Junior MS, Guimarães PTG \& Alcântara EM (2011) Capacidade de suporte de carga e umidade crítica de um Latossolo induzida por diferentes manejos. Revista Brasileira de Ciência do Solo, 35:115-131.

Assis SV, Sediyama GC, Vianello RL \& Martyn PJ (1989) Probabilidade de ocorrência de dias trabalháveis com tratores agrícolas em Uberaba, Minas Gerais. Revista Ceres, 36:299-316.

Ataíde LT, Caramoni PH, Ricce WS, Silva DAB \& Souza JRP(2012) The probability of potentially useful work days during the year in Londrina. Revista Semina, 33:2215-2226.

Aviad Y, Kutiel H \& Lavee H (2009) Variation of Dry Days Since Last Rain as a measure of dryness along a Mediterranean - Arid transect. Journal of Arid Environments, 73:658-665.

Braunack MV, Arvidsson J \& Hakansson I (2006) Effect of harvest traffic position on soil conditions and sugarcane (Saccharum officinarum) response to environmental conditions in Queensland, Australia. Soil and Tillage Research, 89:103-121.

Britto FP, Barletta R \& Mendonça M (2008) Variabilidade espacial e temporal da precipitação pluvial no Rio Grande do Sul: Influência do fenômeno El Niño oscilação Sul. Revista Brasileira de Climatologia, 3/4:37-48.

Cardoso CDV (2005) Probabilidade de ocorrência de deficiência hídrica nos solos da região Central do Estado do Rio Grande do Sul. Tese de Doutorado. Universidade Federal de Santa Maria, Santa Maria. 162p.

Embrapa - Empresa Brasileira de Pesquisa Agropecuária (2006) Centro Nacional de Pesquisa de Solos. Sistema Brasileiro de Classificação de Solos. $2^{a}$ ed. Rio de Janeiro, Embrapa-SPI. 306p.
Embrapa - Empresa Brasileira de Pesquisa Agropecuária (2007) Zoneamento agroclimático do arroz irrigado por épocas de semeadura no estado do Rio Grande do Sul (versão 4). Pelotas, Embrapa Clima Temperado. $37 \mathrm{p}$.

Erthal LC \& Sentelhas PC (1995) Probabilidade da sequência de dias secos em Campinas-SP como subsídio ao planejamento do uso de máquinas agrícolas. In: Congresso Brasileiro de Agrometeorologia, Campina Grande. Anais, Sociedade Brasileira de Agrometeorologia. p. $409-411$.

Fernandes HC, Souza AP \& Vitória EL (2000) Determinação do número de dias potencialmente úteis parta o trabalho com máquinas florestais. Revista Árvore, 24:269-274.

Hemmat A, Tahmasebi M, Vafaeian M \& Mosaddeghi MR (2009) Relationship between pre-compaction stress and shear strength under confined and semi-confined loadings for a sandy loam soil. Biosystems Engineering, 102:219-226.

Hunt D (2008) Farm Power and Machinery Management. 10ª ed. Iowa, Waveland Press. 368p.

Kaufmann V, Castro NMR \& Pinheiro A (2012) Escoamentos superficiais e de drenagem em solo com diferentes manejos e intensidades de chuvas simuladas. Revista Brasileira de Recursos Hídricos, 17:273-285.

Lima Neto JA, Ribeiro MR, Corrêa MM, Souza Júnior VSS, Araújo Filho JC \& Lima JF (2010) Atributos químicos, mineralógicos e micromorfológicos de horizontes coesos de latossolos e argissolos dos tabuleiros costeiros do estado de Alagoas. Revista Brasileira de Ciência do Solo, 34:473-486.

Marchezan E (1994) Arroz vermelho: Caracterização, prejuízos e controle. Ciência Rural, 24:415-421.

Monteiro LA, Sentelhas PC \& Piedade SMS (2014) Dias trabalháveis para o manejo do solo em função da chuva e da disponibilidade hídrica do solo em diferentes regiões brasileiras. Revista Ambiente \& Água, 9:459-475.

Peloia PR \& Milan M (2010) Proposta de um sistema de medição de desempenho aplicado à mecanização agrícola. Revista Engenharia Agrícola, 30:681-691.

Roque AAO, Souza ZM, Araújo FS, Silva GRV (2011) Atributos físicos do solo e intervalo hídrico ótimo de um Latossolo Vermelho distrófico sob controle de tráfego agrícola. Ciência Rural, 41:1536-1542.

Thornthwaite CW \& Mather JR (1955) The water balance. Climatology, 8:01-40.

Rev. Ceres, Viçosa, v. 62, n.4, p. 410-414, jul-ago, 2015 